\title{
Simplified Beam Element Model of Badminton Batting Process Based on Motion Differential Equation
}

\author{
Yingda Li \\ Xianyang Normal University, Xianyang, Shaanxi 712000, China \\ Correspondence should be addressed to Yingda Li; 1311085056@nbu.edu.cn
}

Received 19 April 2021; Revised 12 May 2021; Accepted 17 June 2021; Published 25 June 2021

Academic Editor: Huihua Chen

Copyright (C) 2021 Yingda Li. This is an open access article distributed under the Creative Commons Attribution License, which permits unrestricted use, distribution, and reproduction in any medium, provided the original work is properly cited.

\begin{abstract}
Badminton is not only a traditional sport in China, but also an advantage sport in China. It has good entertainment, appreciation, and exercise, as well as a very broad mass base in China. Badminton racket as the key equipment of badminton, its production and design has a very critical impact on the development of the sport; the current existing research lacks the analysis of badminton batting moment. In order to more intuitively and clearly understand the force and displacement relationship of the racket net at the moment of badminton hitting, this study is based on the differential equation of motion, combined with the finite element analysis method to build a simplified beam element model. In order to verify the reliability of the model, the displacement of the beam element and the rod element of the badminton racket under the same load is constructed. Through the research results, it can be seen that the beam element model is more reliable for the numerical calculation of badminton batting moment. The indepth analysis of badminton batting process has a certain reference significance for the production and design of badminton racket.
\end{abstract}

\section{Introduction}

Badminton first appeared more than 2000 years ago. It was called shuttlecock, Pune, shuttlecock cricket, and so on. Later, it gradually became people's recreational activities and competitive events. Badminton racket was originally made of wood. Modern badminton was first born in England; it was introduced to China in the early nineteenth century. At present, it has become a popular sport among Chinese people. Since the 1980s, China has made a lot of outstanding achievements in international badminton events, and China's badminton level has reached the world's advanced level. From badminton events into the Olympic Games, a total of 18 times it won the championship [1]. Badminton racket as the key equipment of badminton, its performance directly affects the process of badminton hitting. Badminton racket developed from the initial wood materials gradually to bamboo wood mixture, titanium alloy, nanotube materials, etc. The development of badminton racket used in the mesh from the initial sheep intestine gradually developed into nylon, beef tendon, chemical fiber sheep intestine, etc. At present, the badminton racket string used by professional athletes is synthetic string made of chemical fiber sheep intestines. This material not only has very good elasticity, but also is very firm and reliable. However, due to the relatively complex production process of this material, the final cost is high, so it is more used in the use of professional athletes. In this case, there is less research on the racket of nonprofessional athletes, which may cause half the result with half the effort in badminton and even sports injury in serious cases. To know more about the performance of badminton racket and analyze the properties of its materials is conducive to the athletes to better play their own advantages in competitive sports and the popularization and development of the sport [2].

Finite element analysis is a process of solving the essence of a simple problem after it is complicated. It can simulate the load conditions of a real physical system and so on. It is a practical application of mathematical methods. Applying it to the finite element analysis of badminton racket and badminton, it can accurately simulate the working condition, mechanics, displacement changes of the moment when 
badminton ball slaps badminton racket, especially for the analysis of the complex process of hitting badminton, which has the characteristics of high accuracy and wide applicability. As the process of badminton hitting mainly involves displacement and force, the mathematical expression of the relationship between force and displacement can be accurately carried out by using the equation of motion [3]. By combining the finite element analysis method with the differential equation of motion, the mechanical analysis of badminton batting process can clearly show the relationship between the load and displacement in the process of badminton batting [4].

The main contributions of this paper are as follows: firstly, the finite element model of string and beam elements of badminton racket is constructed, which can be used to analyze the micromechanics and displacement of badminton batting process. Secondly, based on the established finite element model, the static numerical calculation of badminton racket net surface is carried out, and the net tension of badminton beam element is simulated.

The innovation of this research mainly has three points: the first is to construct the dynamic differential equation of badminton and carry out the mechanical analysis of the badminton batting process using badminton racket; the second is to construct the badminton net model and verify the correctness and feasibility of the net model through the simulation calculation of beam element and rod element; the third is to analyze the different badminton characteristics and the influence of the tension on the return speed of badminton. This research can let people from a more professional point of view analyze the new badminton batting moment; also it provides a certain theoretical basis for the production of badminton racket. At the same time, it is feasible to apply the finite element analysis method to the process analysis of badminton racket hitting, which also provides a reference for the process analysis of similar sports. From the perspective of theoretical analysis, this paper provides ideas for the finite element analysis of sports equipment. It can be seen that the finite element analysis method can achieve good results in the design and optimization of sports equipment.

\section{Related Work}

Badminton and other high-altitude sports may cause certain sports injury because of their large range of upper limb joint movement. The research on the mechanical properties of badminton batting movement has a positive significance for the in-depth understanding of the physiological analysis in the process of batting. There are great differences in neurophysiology between athletes and nonathletes in the process of badminton batting. Athletes have a very significant functional adaptation in the brain and a very prominent performance in the visual motor response. In the process of badminton, the knee joint and ankle joint are easy to be injured. In sports training and exercise, muscle fatigue is a very common physiological phenomenon. Huang et al. constructed an equivalent multilayer nonuniform threedimensional finite element model of human arm, which can be used to analyze the changes of muscle resistance of muscle fatigue in badminton, basketball, and other sports [5]. In badminton, badminton racket is one of the key sports equipment. Che Hassan et al. developed a detailed substandard for the use of badminton racket, classified the performance, physical performance, chemical performance, and environmental performance of badminton racket, and finally determined carbon fiber as the main production material of badminton racket [6]. Badminton rackets have a very direct and important impact on the overall action and effect of players. Different badminton players also have certain differences in their love psychology for badminton. Shotaro and others proposed a new antihuman robot, which designed a four-degree-of-freedom badminton robot arm and used pneumatic energy to hit the ball skillfully. The design and research of the robot played a positive role in promoting the mature manufacture of antihuman badminton robot [7].

In modern industrial applications, the finite element analysis method is also widely used. Based on the finite element analysis software, Bhelsaikar et al. designed a new type of continuous manipulator. Through the finite element analysis software, it is helpful for the prototype manufacturing and testing of robot gripper [8]. Badia et al. proposed an enhanced finite element space technology based on cell focusing technology to solve the ill-posed problems in the traditional three kinds of nonadaptive finite element technology and obtained the optimal finite element solution by dividing different elements [9]. Hiptmair et al. proposed a finite element method that can be applied to incompressible fluid dynamics equations. The second-order semi-implicit time stepping method is used to establish the energy law. Through numerical verification, the correctness of theoretical prediction and the effectiveness of preprocessor of the method are also illustrated [10]. Wang et al. carried out finite element analysis on the manufacturing process of dielectric materials in electromagnetic engineering. Due to the comprehensive influence of processing technology, environmental temperature, and personnel operation, there are many spatial uncertainties in the manufacturing of dielectric materials in electromagnetic engineering. After introducing the interval field model, the uncertainty of this material can be described, and the upper and lower bounds of electromagnetic response can be calculated by using the interval finite element method [11]. Spherical material is difficult in engineering design. The design of circular material plate by finite element analysis is helpful to test and measure with finite element analysis software, so as to understand the stiffness of the designed material [12]. Karthikeyan and Chandru analyzed the wave propagation of annular elliptic thin plate with different voltage materials. By compiling the computer program of finite element method, the numerical calculation of different shell elements was carried out. The model is simple and effective and is suitable for the material design problems that need to be solved accurately [13]. Andena et al. constructed a three-dimensional finite element model of track and field runway and carried out quasistatic compression test on laboratory samples through the model, so as to continuously adjust the parameters of the model, in 
order to continuously adjust and improve the vibration reduction performance of track and field runway [14]. Yang and others have carried out risk assessment on the training process of badminton players, designed and constructed a platform of Internet of things to analyze the badminton process, and improved the badminton training skills according to the results of the study [15].

From the literature research related to badminton, racket design, and finite element analysis method, we can see that the current research on badminton is more from the analysis of movement and the physiological process of athletes; even the research on badminton is from the material design of badminton; the finite element analysis design is in the engineering design and manufacturing. It can achieve very good results. To sum up, this paper proposes to use the finite element analysis method to build the finite element model of badminton and analyze the mutual mechanics and displacement relationship between badminton and badminton racket based on the motion equation, so as to determine the relevant material parameters of badminton and carry out the relevant mechanical verification.

\section{Construction of Basic Finite Element Model of Badminton Batting Process Based on Motion Differential Equation}

\subsection{Simplified Finite Element Model of Badminton Based on} Motion Differential Equation. The batting moment of badminton is a very short process. The contact time between racket and ball is defined as batting time. The speed before the contact between racket and badminton is the initial speed. The speed after the contact is the return speed. The ratio of return speed and initial speed is the speed ratio of batting. In the construction of the finite element model, the stiffness of badminton, racket cable tension, and axial stiffness need to be considered. The vibration equation of the badminton can be simplified as one of the following equations:

$$
m \ddot{x}(t)+k x(t)=0 .
$$

In the badminton racket model, the stiffness perpendicular to the net is assumed to be nonlinear, so the displacement along the direction perpendicular to the net is also nonlinear. Let $a, b, c$ be three undetermined coefficients, the vertical displacement of the central point of the mesh is $y$, and the vertical force on the central point of the mesh is $f_{s b}$; then, the formula of vertical displacement in the mesh direction can be obtained:

$$
f_{s b}=a y^{3}+b y^{2}+c y .
$$

In order to simplify the model, the impact point of the net surface is regarded as the centroid, and the net surface of the racket is a rigid body. The simplified mechanical model of batting can be obtained from Figure 1, as shown in Figure 2 . The racket can be regarded as a combined system of linear spring and mass. In the process of badminton and spherical impact, it is a process of storing the hitting energy

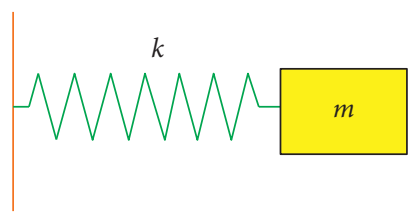

Figure 1: Single degree of freedom system.

in the racket net, which will make the ball and badminton racket vibrate.

Using Newton's second law, we can transform the badminton motion differential equation:

$$
m_{b} \ddot{y}_{b}+k_{b}\left(y_{b}-y_{i}\right)=0 \text {. }
$$

During the process of badminton catching, the impact of badminton on the racket is the resilience of the tennis surface of the racket; thus, we can get

$$
k_{b}\left(y_{b}-y_{i}\right)=f_{s b} \text {. }
$$

Suppose that the velocity of the badminton player is $v_{b_{0}}$, the displacement of the ball is $y_{b}$, and the displacement of the contact point between the badminton and the racket is $y_{j}$ :

$$
\left\{\begin{array}{l}
\dot{y}_{b}(t=0)=v_{b_{0}}, \\
\dot{y}_{j}(t=0)=v_{b_{0}}, \\
y_{b}(t=0)=0, \\
y_{j}(t=0)=0 .
\end{array}\right.
$$

3.2. Parameter Determination of Finite Element Model of Badminton and Racket. The mechanical properties of four common badminton rackets are shown in Table 1.

In Table 1, the initial modulus of fiber refers to the linear stress-strain ratio of the initial part of the load elongation curve of the fiber used in badminton racket. In order to facilitate the calculation, four kinds of badminton racket samples are converted according to the unified diameter of $0.7 \mathrm{~mm}$, from which the calculation parameters of the model can be obtained (see Table 2 for details).

Thirdly, the tension of badminton racket net is selected to represent the tightness of racket line. Different people have different preferences and applicability for the ball line weight. The specific corresponding relationship and use characteristics are shown in Table 3. Among them, professional players are more suitable for medium- and highpound tension rackets. This type of racket has better ball control effect and can clearly feel the control of their own strength on the ball.

For professional badminton players, the racket they use in international competitions must meet the use requirements; that is, the circumference of the racket is not more than $68 \mathrm{~cm}$, the width is within $23 \mathrm{~cm}$, the chord length is $28 \mathrm{~cm}$, and the width is within $22 \mathrm{~cm}$. According to the requirements of badminton racket in international competitions, a finite element model of $28 \mathrm{~cm} \times 22 \mathrm{~cm}$ elliptical badminton racket mesh beam element is defined again. 


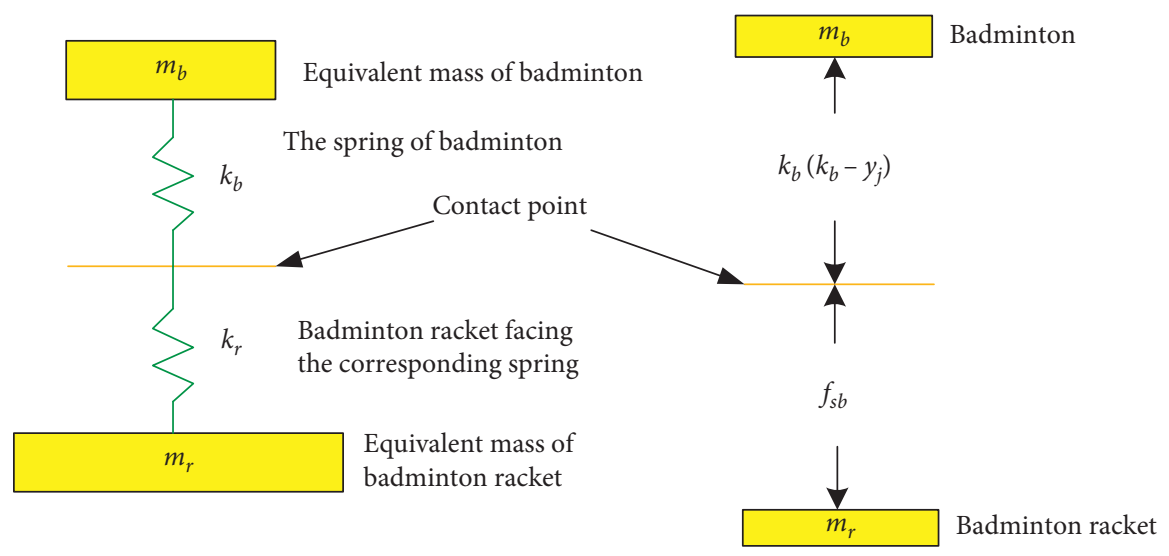

FIGURE 2: Simplified model of force analysis in badminton batting process.

TABLE 1: Mechanical properties of four badminton rackets.

\begin{tabular}{lccccc}
\hline Sample number & Linear density $(\mathrm{g} / \mathrm{km})$ & $\begin{array}{c}\text { Specific strength } \\
\left(\mathrm{Cn} * \mathrm{~g} / \mathrm{km}^{-1}\right)\end{array}$ & $\begin{array}{c}\text { Initial modulus } \\
\left(\mathrm{Cn} * \mathrm{~g} / \mathrm{km}^{-1}\right)\end{array}$ & Maximum strength (N) & Elongation after fracture (\%) \\
\hline Sample 1 & 441 & 51.8 & 173.5 & 228.4 & 43.1 \\
Sample 2 & 472 & 51.8 & 112.1 & 244.5 & 45.5 \\
Sample 3 & 527 & 36.1 & 115.6 & 190.0 & 45.6 \\
Sample 4 & 531 & 24.0 & 114.9 & 127.3 & 46.6 \\
\hline
\end{tabular}

TABLE 2: Ball line calculation parameters of four badminton rackets.

\begin{tabular}{lccc}
\hline Sample number & Mass density & Young's modulus & Poisson's ratio \\
\hline Sample 1 & 1380 & $1.6 e 9$ & 0.374 \\
Sample 2 & 1370 & $1.6 e 9$ & 0.376 \\
Sample 3 & 1227 & $1.4 e 9$ & 0.376 \\
Sample 4 & 1146 & $1.9 e 9$ & 0.378 \\
\hline
\end{tabular}

TABLE 3: The pull tension and use characteristics of badminton racket with different pounds.

\begin{tabular}{|c|c|c|c|}
\hline Pound rating & Pounds & $\begin{array}{c}\text { Tension of stay } \\
\text { wire }\end{array}$ & Use characteristics \\
\hline Low weight & $\leq 20$ & $\leq 90$ & $\begin{array}{l}\text { The racket line is very loose, and the ball has a significant residence time on the racket. It is } \\
\text { suitable for pulling and hanging the ball. }\end{array}$ \\
\hline \multirow{3}{*}{$\begin{array}{l}\text { Medium and low } \\
\text { pounds }\end{array}$} & 21 & 94.5 & \multirow{3}{*}{$\begin{array}{c}\text { Good elasticity, a little sense of retention, attack power will be weakened, suitable for } \\
\text { amateurs }\end{array}$} \\
\hline & 22 & 99.0 & \\
\hline & 23 & 103.5 & \\
\hline \multirow{2}{*}{ Medium pound } & 24 & 108.0 & \multirow{2}{*}{ Amateurs use the most, can play a better performance, and has a significant high elasticity } \\
\hline & & 12.5 & \\
\hline \multirow{2}{*}{$\begin{array}{l}\text { Medium high } \\
\text { pound }\end{array}$} & 26 & 117.0 & \multirow{2}{*}{$\begin{array}{l}\text { It is hard to pull the back court, and it is weak to hit the ball. It is very comfortable to } \\
\text { control the ball. When the strength is large, it can have a very fast speed and can accurately } \\
\text { feel the size of its own strength. }\end{array}$} \\
\hline & 27 & 121.5 & \\
\hline Big pound & $\geq 28$ & $\geq 126$ & $\begin{array}{l}\text { It is easy for professional athletes to control the ball and ensure the match between } \\
\text { strength and speed }\end{array}$ \\
\hline
\end{tabular}

Badminton is divided into feather parts and ball heads. In general, we classify badminton into two categories: plastic and cork according to the materials used in badminton heads. Badminton balls can also be divided into rigid plastics and foam plastics. Cork badminton can be divided into three categories: cork, composite cork, and regenerated cork. Recreational badminton, which takes into account the cost of operation, is usually made of rigid plastics and foam badminton heads. This study adopts composite- and corktype badminton heads.

Compared with the two kinds of badminton head, the use of composite head is not easy to exercise, and the cost is relatively low, but if the use of greater strength to hit the ball, it is easy to break up the lower part of the cork and affect the hitting performance. For beginners, because the hitting power of beginners is not too big, it is not easy to cause 
damage to badminton. At this time, the damage is more concentrated on the feather of badminton. At present, there is no unified classification and numbering standard for badminton feathers, which can be divided into four grades according to the quality of feathers, small hair, medium hair, and large hair according to the type of badminton feathers, and can also be classified according to the thickness of hair stalk and the thickness of hair piece. No matter what the type of feathers is, badminton uses 16 real feathers. The more consistent the feathers are, the better the flying quality of badminton is. High-quality badminton basically uses highquality goose feathers. According to the definition of badminton rules in badminton competition, the height of badminton is $68-78 \mathrm{~mm}$, the diameter is $58-68 \mathrm{~mm}$, and the weight of badminton is $4.74 \mathrm{~g}-5.5 \mathrm{~g}$. The specific parameters are diameter $26 \mathrm{~cm}$, Young's modulus $10.9 \mathrm{GPa}$, Poisson's ratio 0.47 , density $544 \mathrm{~kg} / \mathrm{m}^{3}$, and the overall shape of tetrahedron.

\section{Construction of Net Beam Element Model of Badminton Racket}

4.1. Construction of Differential Equations of Motion for Beam Element and Bar Element. In order to build the basic model of badminton racket beam element mesh, based on the previous relevant parameters of badminton, comparative experimental analysis of bar element and beam element is carried out. According to the definition of mechanics of materials, the relationship between force and displacement of a single string in a badminton racket is shown in the following equation:

$$
F=\left(\frac{\Delta}{l}\right)^{3} E A .
$$

In equation (6), $F$ is the load value of the middle point of the chord, and the value is $1 n ; A$ is the cross-sectional area of the chord, and the value is $3.85 * 10^{-7} \mathrm{~m}^{2} ; E$ is $1.4 \mathrm{GPa} ; l$ is $1 /$ 2 chord length, and the value is $0.1 \mathrm{M}$; and $\Delta$ is the displacement of the middle point of the badminton racket in the load displacement. The formula regards the stress of a single badminton racket as static load. On this basis, the beam element and rod element of the badminton racket can be selected for comparative analysis. The stress analysis is shown in Figure 3.

\subsection{The Finite Element Model Construction of the Net Beam} Element and the Rod Element of Badminton Racket. The displacement of the nodes at both ends of the chord line of badminton racket is limited. Under the setting of each parameter in equation (6), 40 beam element finite element models are constructed and numerically calculated. The results of numerical calculation are compared with the analytical results calculated by the formula. The results are shown in Table 4.

Further analysis from Figure 4 shows that when the load value is $1 n$, the error between the analytical solution and the numerical solution is the smallest, which is $1.06 \%$. The beam element does not change completely in the process of force,

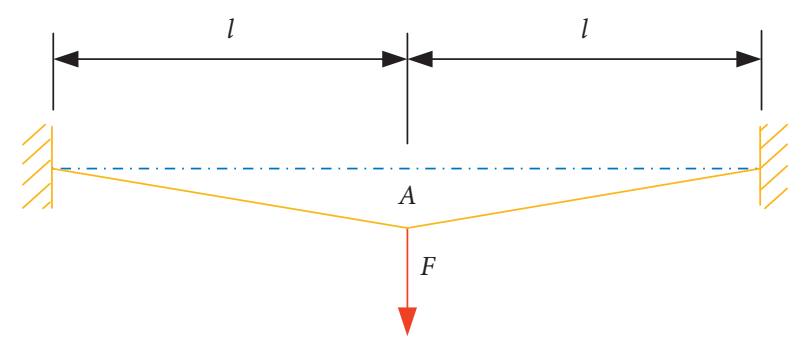

FIGURE 3: Force analysis of single string in badminton racket.

which shows that it is feasible to analyze the beam element when the chord line of badminton racket is thin.

The displacement of nodes at both ends of badminton chord is limited, and $1 n$ load is applied at the midpoint of chord. Referring to the previous parameters, let $F$ be $1 n, E$ be $1.4 \mathrm{GPa}, A$ be $3.85 \times 10^{-7} \mathrm{~m}^{-2}$, and $L$ be $0.1 \mathrm{~m}$. When using the finite element method for analysis, considering the lack of a load balance force in the $y$-direction, a constraint with minimal stiffness can be selected, and the error comparison of springs with different stiffness can be carried out according to the spring stiffness values of $0.1,1,2,3$, and 10 . The results are shown in Figure 5.

It can be seen from Figure 5 that when the stiffness value is 2 , the midpoint displacement value is close to the value of the analytical solution. It can be seen that the construction of the model can be applied to the numerical calculation of badminton net. Therefore, the finite element software is used to carry out the comparative analysis between the load displacement of the badminton club element and the analytical solution. The calculation results are shown in Table 5.

It can be seen from Figure 6 that the displacement numerical solution and analytical numerical solution of the badminton racket bar element are similar to the simulation results of the beam element. When the load value is $1 n$, the error value is the smallest.

From the simulation analysis of beam element and bar element, it can be seen that the error values of single-chord simulation results of badminton are in a small level, so it is necessary to compare and analyze the numerical calculation of badminton racket net surface of beam element and bar element.

\section{Model Construction and Numerical Results of Beam Element and Bar Element}

5.1. Static Numerical Calculation of Badminton Racket Net. The racket line of badminton is a state of overlapping. In the moment of hitting, some net lines on badminton racket will be separated, which makes the net line no longer interact effectively. In order to make the process analysis more close to the real situation, it is necessary to consider releasing some node constraints when building the finite element model. An elliptic finite element model with long axis and short axis of $28 \mathrm{~cm}$ and $22 \mathrm{~cm}$ is constructed. 430 intersections are set up. A position near the middle of racket is selected as the hitting point, then 19 nodes will be constrained to release, and the rest 411 intersections will have 
TABLE 4: Comparison between numerical solution and analytical solution of single string beam element of badminton racket.

\begin{tabular}{lccc}
\hline Load value $(n)$ & Analytical solution of displacement $(\mathrm{mm})$ & Numerical solution of displacement $(\mathrm{mm})$ & Error value $(\%)$ \\
\hline 0.0000 & 0.00 & 0.00 & 0.00 \\
0.0156 & 3.07 & 2.81 & 8.47 \\
0.0312 & 3.86 & 4.61 & 6.48 \\
0.0469 & 4.42 & 4.81 & 4.98 \\
0.0703 & 5.06 & 5.52 & 4.94 \\
0.1055 & 5.81 & 6.42 & 3.99 \\
0.1582 & 6.62 & 7.41 & 2.02 \\
0.2373 & 7.61 & 8.51 & 2.63 \\
0.3560 & 8.72 & 9.78 & 1.81 \\
0.5339 & 9.96 & 11.27 & 1.49 \\
0.8009 & 11.44 & 12.18 & 1.06 \\
1.0000 & 12.31 & &
\end{tabular}

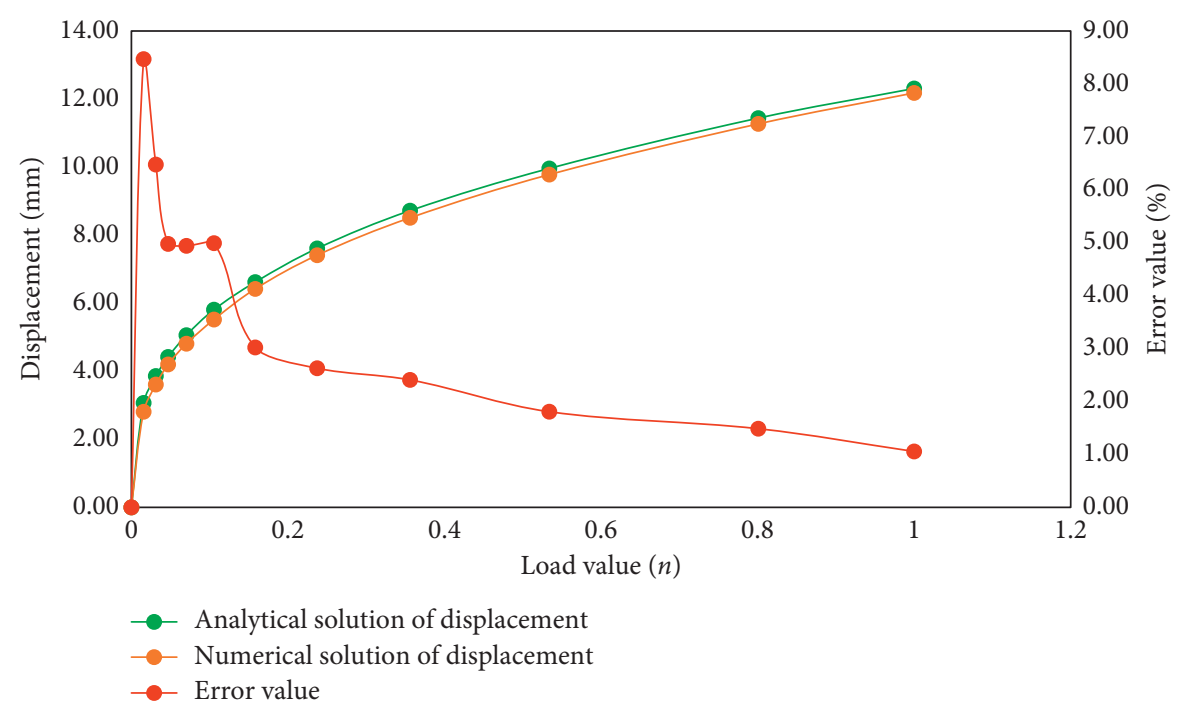

FIGURE 4: Load-displacement curve and error comparison diagram of single beam string element.

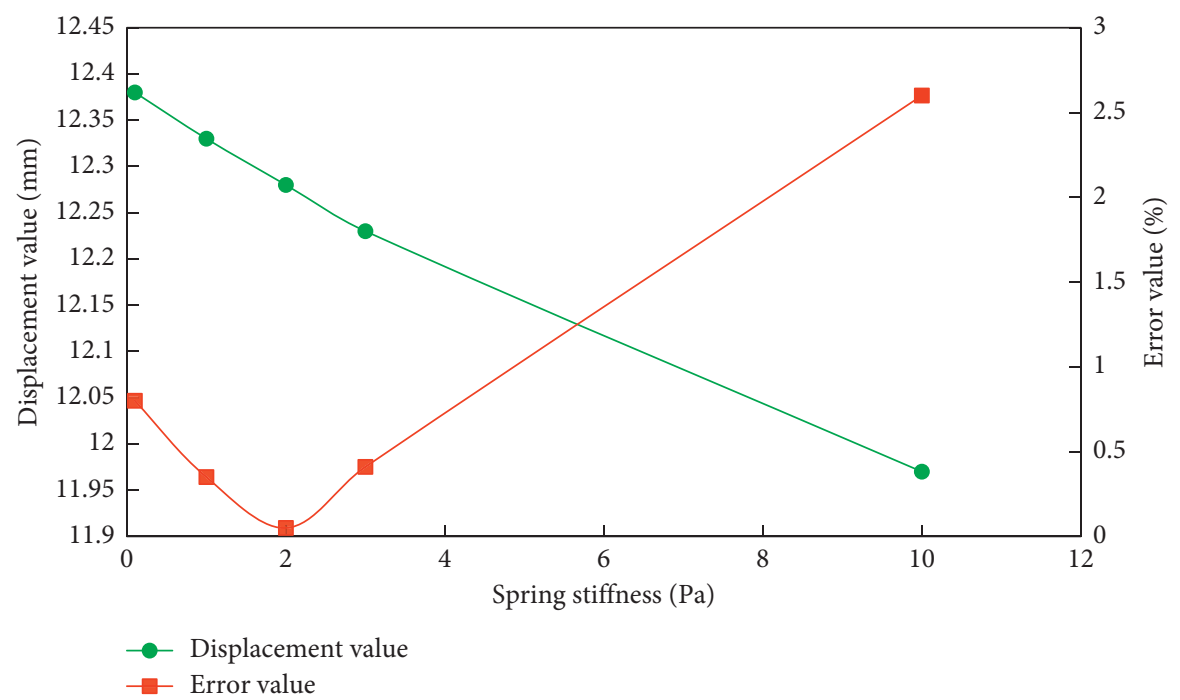

FIgURE 5: Comparative analysis of spring displacement and error with different stiffness.

the force effect. In order to compare the energy value of beam element and rod element, some nodes are deleted to compare the displacement of badminton mesh. The maximum $50 \mathrm{~N}$ load is applied to the mesh surface. The displacement comparison of badminton net surface is shown in Table 6: 
TABLE 5: Comparative analysis of numerical solution and analytical solution of unit point displacement of badminton racket.

\begin{tabular}{lccc}
\hline Load value $(n)$ & Analytical solution of displacement $(\mathrm{mm})$ & Numerical solution of displacement $(\mathrm{mm})$ & Error value $(\%)$ \\
\hline 0.0 & 0.00 & 0.00 & 0.00 \\
0.1 & 5.71 & 5.48 & 7.02 \\
0.2 & 7.14 & 8.11 & 1.03 \\
0.3 & 8.23 & 8.89 & 1.46 \\
0.4 & 9.06 & 9.65 & 1.88 \\
0.5 & 9.76 & 10.29 & 1.13 \\
0.6 & 10.37 & 10.88 & 0.77 \\
0.7 & 10.92 & 11.38 & 0.37 \\
0.8 & 11.42 & 11.85 & 0.35 \\
0.9 & 11.88 & 12.29 & 0.25 \\
1.0 & 12.31 & & 0.16 \\
\hline
\end{tabular}

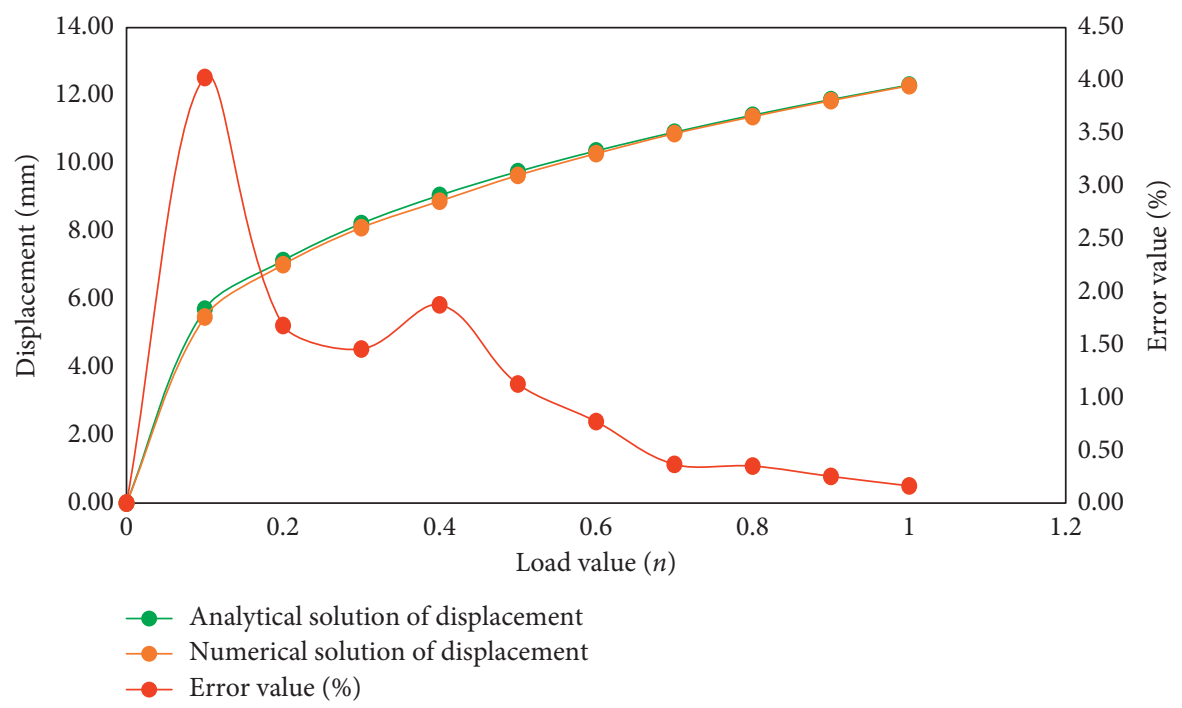

FIGURE 6: Curve comparison of numerical solution and analytical solution of unit point displacement of badminton racket.

TABLE 6: Displacement values of beam element and bar element under different loads.

\begin{tabular}{|c|c|c|c|c|c|}
\hline \multicolumn{3}{|c|}{ Beam element } & \multicolumn{3}{|c|}{ Rod element } \\
\hline Load value $(n)$ & Node not deleted $(\mathrm{mm})$ & Deleted node $(\mathrm{mm})$ & Load value $(n)$ & Node not deleted $(\mathrm{mm})$ & Deleted node $(\mathrm{mm})$ \\
\hline 0.000 & 0.00 & 0.00 & 0.000 & 0.00 & 0.00 \\
\hline 0.196 & 3.84 & 3.86 & 0.101 & 2.36 & 2.36 \\
\hline 0.392 & 5.07 & 5.11 & 0.338 & 4.11 & 4.11 \\
\hline 0.585 & 5.93 & 5.95 & 0.514 & 4.92 & 4.92 \\
\hline 0.874 & 6.87 & 6.93 & 0.768 & 5.86 & 5.86 \\
\hline 1.319 & 7.98 & 8.05 & 1.152 & 6.99 & 6.99 \\
\hline 1.974 & 9.25 & 9.29 & 1.712 & 8.22 & 8.22 \\
\hline 2.967 & 10.69 & 10.78 & 2.547 & 9.67 & 9.67 \\
\hline 4.451 & 12.51 & 12.55 & 3.848 & 11.34 & 11.34 \\
\hline 6.684 & 14.19 & 14.23 & 5.772 & 13.15 & 13.15 \\
\hline 10.024 & 16.28 & 16.37 & 12.978 & 17.62 & 17.62 \\
\hline 15.472 & 18.64 & 18.76 & 19.457 & 20.14 & 20.14 \\
\hline 22.547 & 21.44 & 21.49 & 29.214 & 23.45 & 23.45 \\
\hline 33.724 & 24.57 & 24.63 & 43.175 & 26.93 & 26.93 \\
\hline 50.000 & 28.11 & 28.15 & 50.000 & 28.17 & 28.17 \\
\hline
\end{tabular}

The displacement curve of beam element and rod element with some nodes deleted and nodes not deleted are drawn respectively, as shown in Figure 7.
In Figure $7, \mathrm{~A}$ and $\mathrm{B}$, respectively, represent the displacement curves of some nodes not deleted or deleted in the beam element; $\mathrm{C}$ and $\mathrm{D}$, respectively, represent the 


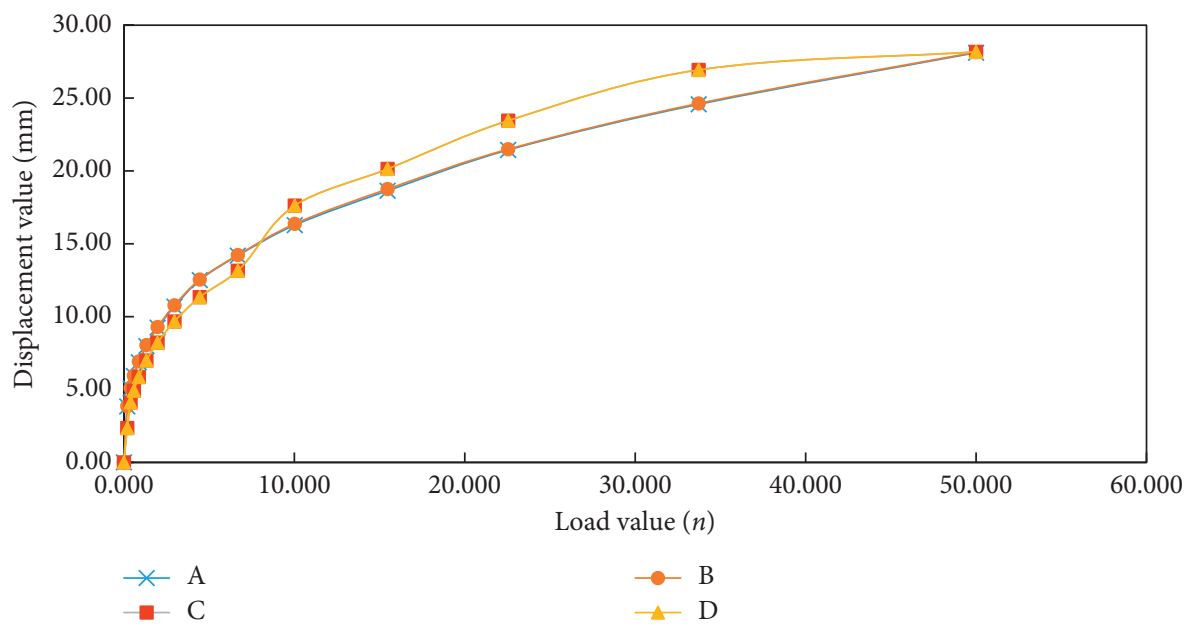

Figure 7: Displacement curves of beam element and bar element with and without deleted nodes.

TABLE 7: Comparison table of numerical calculation of beam element and bar element under different tension of stay wire.

\begin{tabular}{lccccc}
\hline $\begin{array}{l}\text { Tension of stay } \\
\text { wire }(\mathrm{N})\end{array}$ & $\begin{array}{c}\text { Analytical solution of } \\
\text { displacement }(\mathrm{mm})\end{array}$ & $\begin{array}{c}\text { Displacement of beam } \\
\text { element }(\mathrm{mm})\end{array}$ & $\begin{array}{c}\text { Error value } \\
(\%)\end{array}$ & $\begin{array}{c}\text { Displacement of rod } \\
\text { element }(\mathrm{mm})\end{array}$ & $\begin{array}{c}\text { Error value } \\
(\%)\end{array}$ \\
\hline 0.108 & 12.14 & 12.06 & 0.66 & 12.01 & 1.07 \\
0.524 & 11.74 & 11.68 & 0.51 & 1.63 \\
1.054 & 11.18 & 11.14 & 0.36 & 1.04 & 1.25 \\
2.134 & 10.15 & 10.12 & 0.30 & 7.29 & 1.28 \\
5.347 & 7.42 & 7.35 & 0.94 & 4.31 \\
10.248 & 4.48 & 4.47 & 0.22 & 3.79 \\
\hline
\end{tabular}

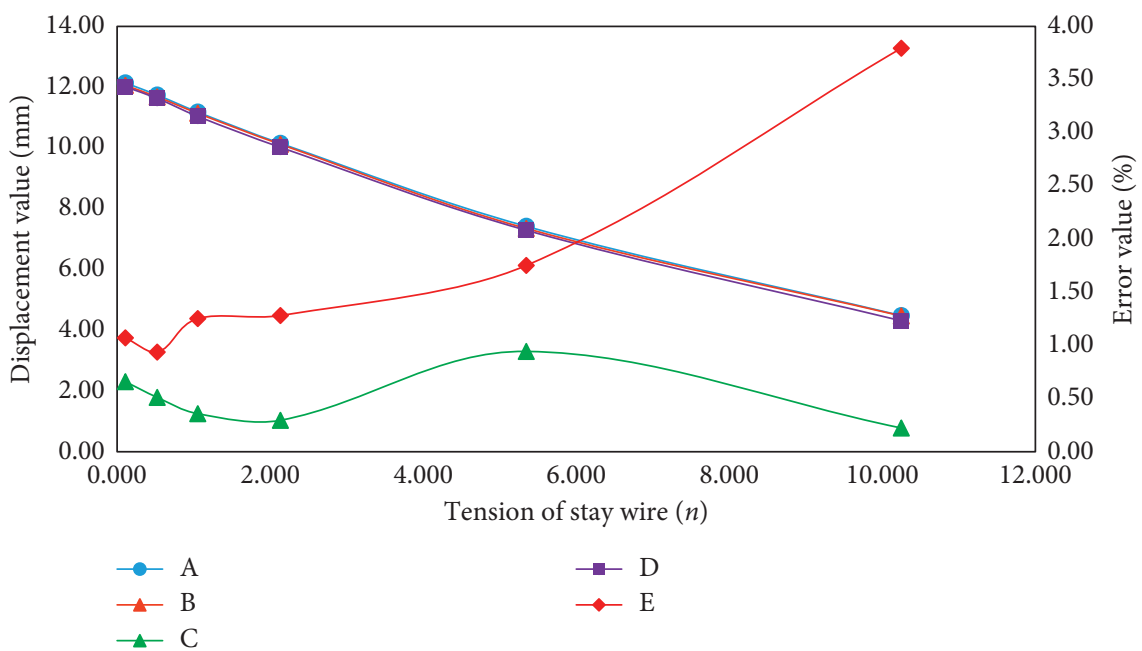

FIGURE 8: Simulation results of net tension of badminton beam element.

displacement curves of some nodes not deleted or deleted in the rod element. Through the comparative analysis of Figure 7 , it can be seen that after deleting some nodes, the net stiffness of badminton racket is reduced, and the displacement change of chord midpoint is the largest under the same load. Considering that in the moment of badminton hitting, some nodes will not be forced, so in this simulation analysis, the deletion of nodes is more in line with the actual situation. Further comparison of the displacement curve difference after deleting the node shows that the difference between the 
midpoint displacement of the beam element and the bar element is $0.28 \%$, and the difference between the midpoint displacement after deleting the node is $0.43 \%$.

\subsection{Simulation Results of Net Tension of Badminton Beam} Element. The string tension $F_{\gamma}$ is added to the net surface of badminton racket, so we can get the formula of analytical solution as follows:

$$
F=\left(\frac{\Delta}{L}\right)^{3} E A+\frac{2 \Delta F_{\gamma}}{L} .
$$

In the finite element model analysis, the applied load at the middle point is $1 n$, the chord length is $0.2 \mathrm{~m}$, and the value of $E A$ in equation (7) is $539 n$. After fixing the two ends of the chord, observe the displacement curve of the beam element and the bar element model under different tension of the stay wire. The results are shown in Table 7.

The curve of the simulation calculation results of the pull wire tension of badminton beam element is shown in Figure 8. A is the analytical solution of displacement, B and C are the displacement value and error value of beam element, respectively, and $\mathrm{D}$ and $\mathrm{E}$ are, respectively, the displacement value and error value of the rod element. According to the displacement curve of the pull tension, it can be seen that with the increasing tension of the cable applied, the displacement value of the midpoint of the chord line on the badminton racquet net surface decreases gradually, which indicates that the displacement of the action point of the mesh surface will be reduced gradually under the same position and the same load. That is, when a constant force is applied to the chord, the completeness near the midpoint position is the lowest.

The error of beam element is the smallest under different tension, so it is more reasonable to use beam element for numerical simulation.

\section{Conclusion}

Applying the finite element analysis method to the simulation of badminton racket net line, the tetrahedral element of badminton can be simulated by combining the differential equation of motion and beam element, so as to deduce the dynamic balance equation in the process of badminton hitting. In order to verify the availability of the model, this study carried out the finite element simulation analysis on the beam element and rod element of badminton racket, respectively. Through the analysis and comparative analysis with the mechanics of materials, it can be seen that the error generated by the beam element and rod element model is small, so it can be applied to the simulation of badminton racket. Based on the simulation model and related parameters, the pull tension is introduced into the string of badminton racket. Through the comparative analysis of the results of static numerical calculation and material mechanics analysis, it can be seen that the error of using beam element is smaller. The final analysis can be seen that with the increasing tension of the stay wire, the midpoint displacement of the badminton racket net model is gradually decreasing. The purpose of this study is to build a usable finite element model of badminton racket hitting and to analyze the mechanics and displacement of badminton hitting process based on this model, which can provide a theoretical basis for the theoretical research of badminton hitting process and the manufacturing of badminton racket and badminton. However, this research is limited to comparative analysis, only limited to the results of finite element analysis and analytical calculation based on mechanics of materials. The follow-up research can be based on the actual operation of professional athletes for comparative analysis.

\section{Data Availability}

The data used to support the findings of this study are available from the corresponding author upon request.

\section{Conflicts of Interest}

The author declares that there are no conflicts of interest regarding the publication of this paper.

\section{References}

[1] R. Abedanzadeh, K. Becker, and S. Mousavi, "Both a holistic and external focus of attention enhance the learning of a badminton short serve," Psychological Research, vol. 17, pp. 1-9, 2021.

[2] M. Naoto and K. Koji, "Comparison of modular control during smash shot between advanced and beginner badminton players," Applied Bionics \& Biomechanics, vol. 2018, Article ID 6592357, 6 pages, 2018.

[3] R. W. L. Lee, C. Y. Andy, and T. W. L. Wong, "Application of analogy in learning badminton among older adults: Implications for rehabilitation," Motor Control, vol. 23, no. 3, pp. 384-397, 2019.

[4] M. Á. Gómez, F. Rivas, A. S. Leicht, and J. M. Buldú, "Using network science to unveil badminton performance patterns," Chaos, Solitons \& Fractals, vol. 135, Article ID 109834, 2020.

[5] L. K. Huang, L. N. Huang, Y. M. Gao et al., "Electrical impedance myography applied to monitoring of muscle fatigue during dynamic contractions," IEEE Access, vol. 99, p. 1, 2020.

[6] M. F. Che Hassan, M. U. Rosli, and M. A. Mohd Redzuan, "Material selection in a sustainable manufacturing practice of a badminton racket frame using Elimination and Choice Expressing Reality (ELECTRE) Method," Journal of Physics: Conference Series, vol. 1020, Article ID 012012, 2018.

[7] S. Mori, K. Tanaka, S. Nishikawa, R. Niiyama, and Y. Kuniyoshi, "High-speed and lightweight humanoid robot arm for a skillful badminton robot," IEEE Robotics \& Automation Letters, vol. 3, no. 3, pp. 1727-1734, 2018.

[8] A. H. Bhelsaikar, D. Roy, and V. Atpadkar, "Estimation of dynamic characteristics of a novel non-parallel detachable-jaw robotic gripper using finite element method," Journal of Physics: Conference Series, vol. 1831, no. 1, Article ID 012010, 2021.

[9] S. Badia, F. Verdugo, and A. F. Martín, "The aggregated unfitted finite element method for elliptic problems," Computer Methods in Applied Mechanics and Engineering, vol. 336, pp. 533-553, 2018.

[10] R. Hiptmair, L. Li, S. Mao et al., "A fully divergence-free finite element method for magnetohydrodynamic equations," 
Mathematical Models and Methods in Applied Sciences, vol. 28, no. 4, pp. 659-695, 2018.

[11] Z. Wang, C. Jiang, B. Ni, C. Wang, J. Zhong, and T. Fang, "An interval finite element method for electromagnetic problems with spatially uncertain parameters," Science China Technological Sciences, vol. 63, no. 1, pp. 25-43, 2020.

[12] C. Itu, A. Öchsner, S. Vlase, and M. I. Marin, "Improved rigidity of composite circular plates through radial ribs," Proceedings of the Institution of Mechanical Engineers, Part L: Journal of Materials: Design and Applications, vol. 233, no. 8, pp. 1585-1593, 2019.

[13] S. Karthikeyan and S. Chandru, "Linear modal analysis of ring shaped-elliptical plate using finite element method Linear modal analysis of ring shaped-elliptical plate using finite element method," Journal of Physics Conference Series, vol. 1597, no. 1, Article ID 12041, 2020.

[14] L. Andena, S. Aleo, F. Caimmi et al., "Modelling the cushioning properties of athletic tracks," Sports Engineering, vol. 21, no. 4, pp. 453-463, 2018.

[15] J. Yang, H. Xia, Y. Wang, and H. Tian, "Simulation of badminton sports injury prediction based on the internet of things and wireless sensors," Microprocessors and Microsystems, vol. 81, no. 1, Article ID 103676, 2021. 